IGDA.5: Supplementary assessment procedures

\section{psychopathological, neuropsychological}

\section{and physical aspects}

IGDA WORKGROUP, WPA

\section{1}

Supplementary assessment procedures may be used to complement the psychiatric diagnostic interview. These include procedures for the comprehensive evaluation of the mental and physical state of the patient (psychosocial procedures are considered in part 6 (IGDA Workgroup, WPA 2003, this suppl.)). Psychopathological assessment instruments, biological tests and procedures and neuropsychological tests are among those used to enrich or refine data collected in a clinical interview in order to arrive at the standardised multi-axial diagnostic formulation

\section{2}

The purposes of these supplementary assessments are:

(a) to accurately identify and describe signs and symptoms in terms of their type, extent and severity;

(b) to increase the reliability and validity of clinical diagnoses, to aid in the process of differential diagnosis, and to identify potential aetiological factors;

(c) to document patients' cognitive skills and limitations for the purposes of estimating capacity for self-care, treatment adherence, and ability to carry out activities of daily living;

(d) to identify particular targets and goals for treatment and rehabilitation and to measure change over time and treatment outcome;

(e) to document psychological and behavioural injuries, rehabilitation potential, and need for treatment; capacity pertinent to legal proceedings;

(f) to support research into mental disorder evaluation, treatment and aetiology.

\section{3}

Various types of supplementary assessment procedures should be considered for use, including self-rated and observer-rated scales, screening tests and scales, symptom and diagnostic checklists, semi-structured and fully structured diagnostic interviews, neuropsychological tests, and biological tests and procedures.

\section{4}

Choice of a supplementary assessment procedure should be based on a number of criteria, including the specific purpose intended (e.g. syndrome identification, symptom severity, clinical change over time); pathological domain (e.g. depression, dementia, personality functioning); characteristics of the patient to be tested (e.g. age, educational level, cognitive status); characteristics of the setting in which the procedure will be used (e.g. primary care practice, psychiatric hospital, community survey); qualifications and training of user (e.g. psychiatrist, general medical professional, lay interviewer); culture of the patient and the evaluator (e.g. first language, translations, concepts of health and illness); psychometric properties of the instrument or procedure (e.g. reliability, validity); cultural validation in the population of interest; and other characteristics (e.g. time frame of interest, time and cost of administration, training required).

\section{5}

Diagnostic checklists and interviews are useful for trained clinicians to ensure that adequate information has been gathered from the patient, informants or other sources, so that a differential nosological diagnosis according to specified diagnostic criteria (as set out in one of the international classifications of mental disorders) can be made.

\section{6}

Brief screening scales or instruments that include exploratory questions and symptom or diagnostic checklists are useful when large numbers of patients or community residents are to be examined for possible mental or physical disorders, with positive cases referred for more extensive evaluation. Instruments that can be administered by a primary care physician or a less-qualified health worker are useful when a mental health clinician is not available. Rating scales for psychopathological symptoms or syndromes identified with Likert or visual analogue scales of frequency or severity are useful to ascertain initial levels of symptom severity and to measure change over time.

\section{7}

The assessment of psychodynamic and cognitive-behavioural perspectives on psychopathology should be considered, through systematic procedures, especially when these assessments are contributory to a better understanding of the clinical condition or to treatment planning.

\section{8}

Neuropsychological assessment helps to ascertain mental and neurological factors and causes of symptoms and disorders and to localise sites of lesions. Neuropsychological tests also appraise cognitive strengths and weaknesses and other behavioural capacities of the patient for the purpose of planning treatment and rehabilitation.

\section{9}

Physical examination and laboratory tests (e.g. of blood or urine) are useful to rule in or out general medical conditions or substances of misuse as a case of mental disorders, and also aid in the diagnosis of other physical conditions or disorders that might affect treatment or clinical management. Brain imaging such as computed tomography or magnetic resonance imaging is helpful in documenting structural and functional brain abnormalities. Electrophysiological tests such as electroencephalography can identify abnormal brainwave activity. All of these procedures help in formulating a more definitive clinical diagnosis.

\subsection{0}

The effective use of supplementary psychopathological, neuropsychological and biological assessment procedures requires appropriate training in their application. The competent interpretation of the results 
requires training in and familiarity with indications for use, applications to particular patient groups and settings, and strengths and weaknesses of the procedures involved.

\section{FURTHER READING}

American Psychiatric Association (2000) Handbook of Psychiatric Measures. Washington, DC: APA
Bech, P. (1993) Rating Scales for Psychopathology. Health Status and Quality of Life: A Compendium on

Documentation in Accordance with the DSM-III-R and WHO Systems. Berlin: Springer.

IGDA Working Group, WPA (2003) IGDA. 6:

Supplementary assessment procedures - functioning, social context, cultural framework and quality of life. British Journal of Psychiatry, 182 (suppl. 45), s50-s51.

Mezzich, J. E., Jorge M. R. \& Salloum, I. M. (1994) Psychiatric Epidemiology: Assessment Methods. Baltimore MD: Johns Hopkins University Press.
Robin, L. N., Wing, J., Wittchen, H.-U., et al (1988) The Composite International Diagnostic Interview. An epidemiologic instrument suitable for use in conjunction with different diagnostic systems and in different cultures. Archives of General Psychiatry, 45, 1069-1077.

Sartorius, N. \& Jança, A. (1996) Psychiatric assessment instruments developed by the World Health Organization. Social Psychiatry and Psychiatric Epidemiology, 31, 55-69. 